Reprinted from

D. Scott Linthicum Nadir R. Farid Editors

Anti-Idiotypes, Receptors, and Molecular Mimicry

(C) 1988 Springer-Verlag New York, Inc.

Printed in the United States of America.

Springer-Verlag

New York Berlin Heidelberg

London Paris Tokyo 
13

\title{
Crime and Punishment in the Society of Lymphocytes: A Speculation on the Structure of the Putative Idiotypic Network
}

\author{
W. Louis Cleveland
}

\section{Introduction}

The clonal selection theory proposed independently by Burnet and Talmage in 1957 envisions the immune system to consist of a sel of antigenspecific lymphocytes that are selected in ontogeny to avoid reactivity with self, leaving only reactivity with foreign antigens $(1,2)$. In this theory, antibodies are viewed in the same light as any other self antigens. Given the absence of self-reactivity, interactions among antibody molecules are not predicted. Jerne, however, appreciated that immunoglobulins are different from other self antigens in that they possess an enormous sequence diversity in their v-regions. This led him to expect the occurrence, under physiological conditions, of internal interactions among v-regions, i.e., idiotype-anti-idiotype (Id-anti-Id) interactions. On the basis of this expectation, Jerne constructed a theory of immune regulation based on a network of interacting v-regions (3).

The assumptions of Jerne's original network theory can be divided into two categories: completeness assumptions and mechanistic assumptions. On the basis of the large v-region diversity, it was assumed that for every paratope in the repertoire, there was a complementary idiotope, and vice versa. It was also assumed that for every foreign epitope there was an internal cross-reactive idiotope, i.e., an "internal image." On the basis of experimental findings, it was postulated that antibodies of different specificity might share idiotopes. In addition to these completeness assumptions, there were mechanistic assumptions that concerned the activating and suppressive qualities of idiotopes and paratopes (3).

The mechanistic assumptions of Jerne's original network theory appear untenable in the light of modern findings (4). However, the completeness assumptions have not been ruled out. Indeed, the internal image concept, which arises naturally from the completeness assumptions, has recently been supported by many experimental findings (5). By themselves, the 
completeness assumptions do not constitute a theory of the immune system. Rather, they demarcate a large class of theories which have these assumptions in common. This is an important consideration since the completeness assumptions in themselves do not allow clear predictions. Only if appropriate mechanistic assumptions are added can definite predictions be derived and tested.

As originally appreciated by Ehrlich, the most basic feature of the immune system is self-nonself (S-NS) discrimination (6). In individuals free of autoimmune disease, there is apparently no aggressive response against S-antigens, whereas aggressive responses against foreign antigens are freely made. It is, therefore, an essential requirement of any theory of the immune system to provide a mechanism by which S-NS discrimination is established. As emphasized by Cohn, S-NS discrimination cannot be encoded in the germline and therefore must be learned by the system in ontogeny (7). As yet, none of the network theories that have been proposed have offered mechanistic assumptions that provide a clear pathway to $S$ NS discrimination. It is the purpose of this communication to explore a set of mechanistic assumptions that may provide such a pathway. In addition, the theoretical construction that is offered suggests a generalization of the veto phenomenon and provides an interpretation of MHC-restricted antigen recognition by $\mathrm{T}$ cells that is based on internal images of $\mathrm{MHC}$ determinants. Also incorporated into the model is the currently emerging paradigm of antigen presentation. A consideration of antigen processing has led to the proposal that the internal image concept be extended to include cross-reactions between processed v-region fragments and fragments of conventional antigens. It is further suggested that $\mathrm{T}$ cells specific for processed v-regions may influence immunoglobulin and T-cell receptor rearrangements.

\section{Ontogeny of S-NS Discrimination in the B-Cell Compartment}

The theory that will be presented in this discussion envisions the idiotypic network to be analogous to a human society. Human societies often can be divided into two segments: one segment that consists of an establishment composed of law-abiding citizens that do not commit crimes against each other and another segment, often referred to as a fringe element, whose members commit crimes against each other and against the establishment. Although there is continual antagonism between the establishment and the fringe element, the establishment, by definition, remains dominant. Within the establishment there is often a relatively stable stratification, with some members having higher rank than others. The mechanistic assumptions that we offer in conjunction with Jerne's completeness assumptions lead to a society of lymphocytes that has analogous properties. 
In the case of the B-cell compartment, we invoke three principles of interaction which are referred to as the principle of symmetrical stimulation, the principle of mutual antagonism, and the principle of self protection. Consider an anti-S B cell expressing a newly emerged specificity. Upon encounter with antigen and an appropriate $T$ helper cell, it will be stimulated to differentiate into a plasma cell and secrete antibody. On the basis of the completeness assumptions, we expect that within the expressed $B$-cell repertoire, there will be another B cell that is anti-Id to the anti-S $B$ cell. The secreted anti-S antibody will bind the membrane receptors of the anti-Id B cell and set in motion a series of events that will lead to Tcell recognition, clonal proliferation, and stimulation of anti-Id secretion. The pathway which leads to the stimulation of the anti-Id B cells is, of course, a matter of critical importance and will be discussed in the section dealing with antigen presentation. What should be taken for granted at this point is that the stimulation of the anti-Id B cell can occur at very low concentrations of Id.

With time, the concentration of Id and anti-Id in body fluids will rise. When the Id and anti-Id concentrations reach sufficient levels, we assume that the clones exert antagonistic effects on each other. The principle of mutual antagonism assumes that the two clones will tend to destroy each other by antibody-dependent mechanisms. These mechanisms could include killing by complement, by cytotoxic cells of several types which possess $\mathrm{Fc}$ receptors that recognize antibody-coated target cells $(8)$, and by cytotoxic $T$ cells that recognize processed v-regions. The latter possibility will be discussed in the section dealing with antigen presentation. If no other factors were involved, one would expect that it would be a matter of chance as to whether the Id or anti-Id clone would win the conflict. However, there is another factor involved. The conflict takes place in a milieu containing the S-antigen for which the Id antibodies are specific. According to the principle of self protection, the presence of Santigens will enable the anti-ld clone to become victorious, provided, of course, that the anti-Id clone is itself not specific for an S-antigen. This is assumed to depend on the ability of S-antigens to adsorb the anti-S antibodies, thereby reducing their concentration and biasing the outcome of the conflict in favor of the anti-Id clone. It must be acknowledged that some S-antigens are sequestered or present at very low concentrations. In these cases, the adsorption may be inadequate to provide protection and other mechanisms may be involved.

The model can be summarized in general terms as follows:

1. There is an Id-anti-Id symmetry in the expression of B-cell receptors.

2. There is a symmetry in the stimulation of Id-anti-Id pairs.

3. There is a symmetrical antagonism between Id-anti-Id pairs that is antibody dependent.

4. When the Id member of an Id-anti-Id pair is anti-S, the symmetry of antagonism is broken by the presence of S-antigen in such a way that the anti-Id clone becomes dominant. 


\section{Spontaneous Development of a B-Cell Establishment}

The formation of a functional immunoglobulin gene requires DNA rearrangements in which $\mathrm{V}, \mathrm{D}$, and $\mathrm{J}$ gene segments are selected and made contiguous by recombination events that remove intervening sequences (9). It appears that the selection of individual segments depends on an inherently stochastic process. This implies that anti-S B cells will continually emerge throughout the life of the organism. The above model predicts that this continual emergence of anti-S clones will lead to frequent stimulation of the clones that are anti-Id to anti-S. Since these clones become dominant, frequent restimulation should therefore lead to an "establishment" of anti-Id clones whose secreted immunoglobulins can be expected to constitute a substantial portion of the immunoglobulins in body fluids. These clones can also be expected to be represented prominently in the pool of memory B cells.

It must be appreciated that there are many S-determinants and that for each of these determinants there will be many different complementary $v$-regions. Associated with each of these v-regions are multiple idiotopes. Clearly, the number of clones that are anti-Id to anti-S is likely to be very large. This means that there will occasionally be opportunities for conflicts among clones that aspire to become members of the establishment. In these cases, the principle of self protection does not apply. Conflicts should therefore proceed until the weaker clones are defeated, yielding a set of clones which do not interact with each other. The absence of interactions within the establishment and its quantitative dominance within the network have the important consequence of restricting the levels of immune complexes that arise as a result of network interactions.

\section{Composition of the B-Cell Establishment}

To analyze further the composition of the B-cell establishment, it is necessary to consider the types of anti-Id antibodies that might be stimulated by anti-S clones. Currently, several different kinds of anti-Id antibodies are recognized to exist. Some anti-Id antibodies recognize extremely private idiotopes (10) whereas others recognize more public idiotopes that are referred to as cross-reactive idiotopes (11). Id-anti-Id interactions may, or may not, be inhibitable by antigen (12). In addition to these types of anti-Id antibodies, which will be referred to as classical anti-Ids, there are also internal image anti-Ids (13) and epibodies (14).

Given that there are different types of anti-Ids, the representation of these types in the establishment must be considered. Membership in the establishment is based on the ability of a clone to destroy anti-S clones and the degree of protection provided by S-antigens. It is immediately apparent that internal images may be especially efficient in the destruction 
of anti-S clones. This follows from the fact that all clones which recognize a particular determinant, regardless of the details of their v-region sequences, should be dominated by a single clone that expresses an internal image of the determinant. This is in contrast to classical anti-Ids that recognize idiotopes that are unique to a particular clone. Many different clones recognizing private idiotopes would be needed to dominate a heterogeneous population of anti-S clones that react with a single S-determinant. The same consideration should apply, although to a lesser extent, to classical anti-Ids that recognize cross-reactive idiotopes.

As yet, there is little evidence supporting the existence of internal images of S-antigens on normal serum immunoglobulins. However, a recent report by Holmberg et al. (15) described the presence of an internal image of a self class II MHC determinant on a TNP-specific hybridoma derived from a normal neonatal spleen. The monoclonal anti-class Il MHC antibody used to detect this determinant also showed reactivity with normal serum from both 12-day-old and adult mice.

In view of the fact that different types of anti-Ids may have very different efficiencies in performing the functions of establishment clones, it seems reasonable to assume that the establishment represents a stratified society in which various members have different ranks. The rank of a clone is likely to depend on the frequency with which complementary anti-S clones emerge in ontogeny, since the stimulation and proliferation of establishment clones will depend on small amounts of anti-S antibodies. Thus, it is clear that internal image clones not only are more efficient in dominating anti-S clones but are also more likely to be stimulated than those expressing classical anti-Ids.

The concept of rank can be defined further. For example, as pointed out by Jerne et al. (13), it is possible that some internal image antibodies can image two or more determinants. The more S-determinants imaged by an establishment clone the higher its rank is likely to be.

Another factor that may affect rank is specificity for foreign antigens. There seems to be no constraint preventing establishment clones from having specificity for foreign determinants. Stimulation of establishment clones by foreign antigens should add to the stimulation provided by the attempted emergence of anti-S clones. The possibility that establishment clones can have specificity for foreign antigens leads to a consideration of epibodies (14).

A basic problem facing the immune system is that foreign antigens often share determinants with S-antigens. There has to be a mechanism which restricts the response to the foreign determinants. A solution is possible within the establishment structure already described. For example, the response to shared determinants could be down-regulated by clones expressing internal images of the shared determinants. These clones could, of course, be distinct from those recognizing the foreign determinants. However, this down-regulation might also be mediated by epibodies which 
simultaneously are specific for the unshared determinant and bear an internal image of the shared determinant. Having both functions in a single structure would seem to offer increased reliability and efficiency in downregulating the undesired clones. Epibody-secreting clones should have a high rank because there are two pathways of stimulation.

\section{Self Ligand-Receptor Systems Represent a Special Case}

A tacit assumption underlying the construction of our theory so far is that S-antigens do not interact with each other. As will become apparent, such an assumption is required for the absence of interactions between internal images in the establishment. However, this assumption is certainly not correct. For example, within the set of S-antigens there are numerous internal interactions, which we shall refer to as ligand-receptor systems.

To analyze the complications introduced by these interactions, it is necessary to consider the prevailing concept of antigenic determinant. Classically, antigenic determinants are structures at the surfaces of macromolecules which are intuitively regarded as "protuberances" (16). This idea is a companion to the notion that the antibody combining site is a cavity. However, a number of laboratories have raised antibodies that appear to be internal images of low-molecular ligands of physiological receptors (5). If the ligands interact with receptor combining sites that are cavities, then it follows that the combining site on the internal image antibody is a protuberance and not a cavity. It would also seem prudent to consider interactions between molecular surfaces that are neither cavities nor protuberances, but which possess complementarity as a result of interdigitating structures. As noted by Erlanger, classical concepts of antibody combining sites are likely to be oversimplified (17).

Once cavities are regarded as antigenic determinants, it becomes natural to regard anti-ligand antibodies as internal images of receptor combining sites. It should be noted that, in some cases, anti-ligand antibodies and physiological receptors have been shown to exhibit similar specificity patterns (18). In the case of a self ligand-receptor system, it seems clear that an internal image of the ligand is an anti-S antibody because of its reactivity with the receptor combining site. However, it is also anti-Id to anti-S, since it should react with an antibody to the ligand. Likewise, the antiligand antibody is also simultaneously anti-S and anti-Id to anti-S.

These considerations, at first sight, appear to suggest that internal images of self ligand-receptor systems should be forbidden from the establishment, since the clones will continually be in conflict and reactive with S-antigens. However, the above argument has tacitly assumed that antibody specificity 
is qualitative in nature. Affinity and valency effects have been neglected. Taking the latter into account, it is possible to conceive of circumstances in which the above difficulties can be avoided.

Consider a low-affinity antibody to a soluble monovalent S-ligand. This antibody, especially if it is an IgM antibody, might have quite high avidity (multivalent affinity) for the membrane immunoglobulins on a B cell bearing internal images of the ligand. Because of the low affinity of the antiligand antibody for soluble monovalent ligand, the ligand image B cell would not be protected. On the other hand, if the physiological receptor is a cell surface receptor, then the ligand image antibodies can bind in a multivalent fashion, leading to high avidity. Hence, anti-ligand B cells will be protected by the physiological receptor from destruction by the ligand image antibodies. This will lead to a dominance of clones producing lowaffinity anti-ligand antibodies, which can be regarded as internal images of the physiological receptor combining site. If the affinity of these antibodies for physiological ligand is sufficiently low in relation to that of the physiological receptor, then no pathology should result.

The above scheme leads to an elimination of ligand images and permits the emergence of low-affinity receptor combining site images. However, it does not provide a mechanism for the elimination of clones that produce receptor combining site images having high affinity for the physiological ligand. Such clones, which might cause autoimmune disease, could, however, be eliminated by anti-Id antibodies of the classical type, since clones producing these antibodies would be protected by the ligand. Thus, even though internal images of receptor combining sites may sometimes belong to the establishment, it appears that the establishment must also contain anti-Ids of the classical type.

\section{Maintenance of Immunological Memory in the B-Cell Compartment}

The linkage within the establishment of specificity for foreign epitopes and anti-idiotypic specificity for anti-S clones creates an interesting possibility for maintaining immunological memory. The important point is that establishment clones specific for foreign epitopes can be stimulated by anti-S Id in addition to foreign antigens. This means that the anti-foreign establishment clones will continue to be periodically stimulated after the foreign antigen has been cleared. These considerations lead to the expectation that the set of B-cell establishment clones is an important repository of long-term memory in the B-cell compartment. That immunological memory could be stored in the idiotypic network is a possibility that was noted by Jerne in his original paper (3). 


\section{Interactions within the Fringe Element}

Within the fringe element, clones may react with each other, with the establishment, and with both self and foreign antigens. Unlike the establishment, the fringe element is characterized by internal conflict. An important consequence of this is that secreted fringe element antibodies should often form immune complexes with other fringe element antibodies or with establishment antibodies and be cleared from circulation. This is expected to make temporary any usefulness in providing protection against pathogens and to reduce reaction with S-antigens. This also means that the persistent titer to a foreign antigen must be produced by establishment clones. As noted previously, long-lived clones of memory cells should also belong to the establishment.

\section{Stability of the Establishment}

As indicated above, establishment antibodies can form immune complexes with fringe element antibodies or with foreign antigens. This means that the stability of the establishment cannot reside in individual molecules. Similarly, individual cells in establishment clones might also be destroyed in conflicts with fringe element clones. The stability of the establishment would therefore have to reside in the longevity of its clones and ultimately in the pattern of specificities that is responsible for the periodic restimulation of these clones. The absence of stability within the fringe element would be a consequence of a continual alteration of the pattern of specificities expressed as a result of internal conflicts and conflicts with the establishment. It should be noted that Jerne has concluded from an analysis of lifetime measurements that it may be possible to divide network components into long-lived and short-lived sets (16).

\section{Autoimmune Disease and the Establishment}

A network consisting of a fringe element and an establishment provides a context in which to recast the traditional perspective of autoimmune disease. First, there is a basic distinction between an autoimmune response and an autoimmune disease. This follows from the fact that, in our model, autoimmune antibodies are normally and usefully produced by fringe element clones. Because of the transient nature and low levels of such antibody production, we assume that clinically significant autoimmune disease does not result. Clinical autoimmune disease is assumed to be a 
consequence of anti-S clones finding their way into the establishment as a result of aberrant regulation. Membership in the establishment should allow long-term production of relatively higher levels of autoimmune antibody. Given the stability of the pattern of specificities expressed in the establishment, one would expect disease-producing antibodies to be part of this stability. This perspective is consistent with the findings for myasthenia gravis. Tzartos and coworkers have found that the specificity pattern of antibodies to the acetylcholine receptor is remarkably constant over periods of many years in spite of therapeutic maneuvers to control the disease (19).

\section{A Liberal or a Conservative Society?}

So far it has been assumed that anti-S clones are destroyed by the establishment. This may not always happen as genomic alterations in v-regions may allow rehabilitation of anti-S clones. There is a growing body of data supporting the occurrence of frequent intraclonal somatic mutations following antigen stimulation (20-22). It also appears that multiple rearrangements can occur (9). Given these processes, it is possible that clones initially expressing anti-S reactivity can undergo genomic alterations that lead to a loss of reactivity with S-antigens and also a loss of incompatibility with the B-cell establishment. This type of mechanism would not only rehabilitate fringe element clones, but would also generate diversity as was originally foreseen by Jerne (23).

\section{Network Interactions Involving the T-Cell Compartment}

It is now known that for major classes of $\mathrm{T}$ cells, antigen recognition is MHC-restricted. For such T cells, antigen must be seen on membranes in association with either Class I or Class II S-MHC molecules [(24) and references therein]. This type of antigen recognition seems fundamentally different from B-cell antigen recognition, which is not restricted to membrane antigens and which does not require association with MHC molecules. It is a matter of major importance to consider whether MHC-restricted T-T interactions are a part of the putative idiotypic network and whether they might obey the interaction principles we have described for the B-cell compartment. However, current knowledge seems inadequate for the construction of a detailed mechanistic model at this time. Nonetheless, it is interesting to consider the possibility that the repertoires of Class I-restricted and Class II-restricted T cells may also contain estab- 
lishments in which the clones express v-regions that bear internal images of S-determinants or specificities that are anti-idiotypic to anti-S T-cell receptors. It is also interesting to consider the interactions that may occur between the $\mathrm{T}$-cell and $\mathrm{B}$-cell repertoires.

\section{Interactions between the B-Cell and T-Cell Compartments}

Let us consider $\mathbf{T}$ cells arising in ontogeny after the B-cell establishment has already formed. If the $\mathrm{T}$-cell receptor $\mathrm{V}$-regions react with establishment immunoglobulins that are in body fluids, then the cells bearing them may be destroyed by the cytotoxic mechanisms outlined previously. This suggests that $\mathrm{T}$-cell receptors must avoid reactivity with establishment immunoglobulins. Avoidance of reactivity can be achieved if T-cell receptors imitate establishment immunoglobulin v-regions, since the latter set of v-regions is itself free of internal interactions. It is therefore clear that the assumption that $\mathrm{T}$-cell receptors bear internal images of $\mathrm{S}$-antigens is consistent with the absence of interactions between soluble establishment immunoglobulins and $\mathrm{T}$-cell receptors. The imitation of immunoglobulin $\mathrm{v}$-regions by $\mathrm{T}$-cell receptors that is sometimes seen in normal mice is absent in mice artificially devoid of B cells (25). In view of these considerations, it seems likely that the B-cell compartment has considerable influence on the idiotypic structure of the T-cell repertoire.

Are there interaction pathways that allow the $\mathrm{T}$-cell repertoire to influence the B-cell repertoire? In the foregoing example, it is assumed that secreted immunoglobulins are already present in body fluids before the $T$ cell expresses its receptor. If soluble immunoglobulin were not present, the $T$ cell could interact directly with the B-cell surface immunoglobulins, and in the case of a cytotoxic $T$ cell, destroy the $B$ cell. Here a native immunoglobulin is recognized (or recognizes) in a non-MHC-restricted fashion. Recent in vitro evidence raises the possibility that such interactions may occur in vivo $(26,27)$. It is also possible that native B-cell idiotopes can be recognized by $\mathrm{T}$ cells in an MHC-restricted fashion (28). This type of interaction might occur even in the presence of soluble Bcell Id, since MHC-restricted recognition is generally thought to be uninhibitable by soluble antigen. However, these examples, which involve the recognition of native immunoglobulin idiotopes, are as yet only formal possibilities. The bulk of available data indicates that $\mathbf{T}$ cells recognize most soluble protein antigens only after they have been processed and therefore denatured. It is possible that in vivo $\mathrm{T}$ cells recognize immunoglobulin $v$-regions only after processing. This possibility will be discussed in detail in the section dealing with antigen presentation. Although detailed predictions cannot be made with available data, it nonetheless 
appears that bidirectional interactions between the T-cell and B-cell repertoires are likely.

\section{T-Cell Receptor Internal Images and the Veto Phenomenon}

Among the more interesting recent discoveries in cellular immunology is the veto phenomenon, which was discovered by Miller and Derry in 1979 (29). It was first observed in studies of the mixed lymphocyte reaction (MLR). It was found that a subpopulation of B-strain cells could suppress an A anti-B MLR. Further studies suggested that these cells, which were referred to as veto cells, have the capacity to suppress Class I-restricted cytotoxic $\mathrm{T}$ lymphocytes (CTL) that recognize any antigens expressed on the veto cells (30). In other words, veto cells have the ability to suppress CTL that attack them. The suppression mechanism appears to be different from the classical T-cell cytolytic mechanism. Efforts to find a soluble suppressor factor have been unsuccessful. Suppression appears to be permanent (30). Veto cells have been found in bone marrow, thymus, and fetal liver of normal mice. In athymic nude mice, bone marrow and spleen contain veto cells. In spleens and lymph nodes of normal mice, veto cells can be found after a period of in vitro incubation (30). Of special interest is the finding that mature cytotoxic $\mathrm{T}$ cells can have veto activity $(31,32)$.

It is clear that the veto phenomenon could play an important role in SNS discrimination. However, according to current thinking, the veto effect is considered to be limited to antigens expressed on the veto cells $(30,33)$. Moreover, the mechanism is regarded to be independent of the antigenMHC specificity of the cell in the case of CTL-type veto cells (33).

The main point of this discussion is to emphasize that the presence of internal images of S-antigens on Class I-restricted T-cell receptors of mature CTL would provide a basis for extending the veto phenomenon to the entire set of S-antigens represented as internal images. In order for this scheme to work, it is necessary that veto suppression be activated when an anti-Id CTL attempts to attack the veto cell through recognition of the T-cell receptor on the veto cell. We are unaware of any evidence against this possibility. On the contrary, Fink and co-workers have found that a clonotypic antibody to the T-cell receptor on a cloned T-cell line augments the veto activity by 300 -fold (34). This augmentation, which may be due, at least in part, to the induction of cell proliferation, suggests that cross-linking of the clonotypic receptor does not interfere with veto activity. Conceivably, cross-linking the clonotypic receptors by receptors on an attacking $\mathbf{T}$ cell might activate veto suppression as it does for other antigens expressed on the veto cell.

As noted by Miller (30), effector T-suppressor cells have been reported 
to bear Ly2 and Class II MHC antigens (35), raising the possibility that Class II-restricted T cells are also subject to veto control.

According to the scheme presented here, mature CTL belonging to the establishment have both dual specificity and dual effector functions. On the one hand, they are specific for targets bearing foreign antigens and can lyse these cells. On the other hand, they bear internal images of Santigens and can suppress CTL having specificity for these S-antigens. These features are directly analogous to the properties postulated for establishment antibodies that have specificity for foreign antigen and antiId specificity for anti-S B-cell receptors.

\section{T-Cell Receptor Internal Images of MHC-Determinants and MHC-Restriction}

The recent extraordinary progress in characterizing the molecular genetics, amino acid sequence, and peptide structure of the T-cell receptor (36-39) has so far failed to clarify the basis of $\mathrm{T}$-cell antigen recognition, which exhibits the phenomenon of MHC-restriction. On the contrary, the new data have intensified the enigma of MHC-restriction. It has been revealed that the T-cell receptor is an antibodylike molecule (40), which is puzzling since antibodies do not exhibit MHC-restriction. In the case of MHCrestricted antigen recognition, antigen must be associated with membranes bearing MHC molecules in order to be recognized. MHC-restriction appears to be a binding event phenomenon in which high-affinity complexes occur only when a ternary complex consisting of the T-cell receptor, antigen, and $\mathrm{MHC}$ is formed $(41,42)$. Binary complexes consisting of antigen and $\mathrm{T}$-cell receptor or $\mathrm{MHC}$ and T-cell receptor do not functionally activate the T-cell and do not lead to adhesion between the T-cell and a cell bearing antigen alone or antigen + incorrect MHC. The problem, therefore, is to understand why both antigen and $\mathrm{MHC}$ are required for a functional binding event and why neither alone will give functional binding. Essentially two types of models have been proposed to account for MHC restriction: dual recognition models which postulate the existence of two sites that recognize independent antigen and MHC determinants and single recognition models which envision a single-site receptor that recognizes a neoepitope which arises upon the formation of a complex between conventional antigen and MHC [(24) and references therein]. As yet, it is not possible to rule out either type of model. Previous formulations of both types of models are essentially "local" interpretations which consider the $\mathrm{T}$-cell receptor or the antigen-MHC complex as an isolated entity. In this discussion, we offer a "non-local" interpretation of MHC-restriction that is inspired by a network perspective. This interpretation represents an extension of an allosteric dual recognition model which was previously proposed by this writer in collaboration with Erlanger (24). The unique 
feature of this model is that the sites for antigen and MHC are not independent but are coupled by an allosteric mechanism. The basic idea is that the binding of $\mathrm{MHC}$ to the $\mathrm{T}$-cell receptor causes a conformational change at a second site, conferring on this site the ability to bind conventional antigen. This leads to the initial formation of a ternary complex, which may, if positive cooperativity occurs, undergo further internal conformational reorganization, to produce a tenary complex of sufficient affinity to trigger the $\mathrm{T}$ cell. This model is, in a sense, a mirror image of a neoepitope model, since it envisions the existence of neoparatopes that arise following interaction with conventional antigen and MHC. It encounters at least two basic difficulties, which we refer to as the dual recognition problem and the neoparatope problem.

\section{The Dual Recognition Problem}

This problem is one that arises with attempts to rationalize MHC-restriction in the context of a two-site T-cell receptor. Its essence is revealed by a consideration of an argument for dual recognition that was recently given by Parham (43). Parham starts his argument by noting that some monoclonal anti-MHC antibodies bind to cells only if they are bivalent. Apparently, the monovalent binding energy is too low to give detectable binding, whereas in the bivalent case the partial additivity of the binding energies of the two sites leads to binding. He then generalizes by imagining an antibody of mixed specificity, one site specific for MHC, the other for conventional antigen. In this way, he accounts for MHC-restriction, i.e., binding is seen only if both species are present. An important point here is that additivity of binding energy occurs only because the two species of antigen molecules are anchored in the same membrane. That is, if antigen and $\mathrm{MHC}$ molecules were in free solution, no additivity of binding energy would occur. This point highlights the fact that Parham's argument applies only to receptor molecules that are in free solution. Let us now suppose that the heterospecific antibodies are anchored in the membrane of a cell and that the number on a single cell may be greater than 10,000. This is the case that is relevant to the T-cell receptor, since the latter is a membrane receptor. Suppose the cell with heterospecific antibodies collides with another cell bearing self MHC and no conventional antigen. Clearly, there will be an opportunity for extensive multivalent interactions between the matrix of anti-MHC sites and the matrix of MHC antigens on the target cell. Thus, the heterospecific cells should adhere to targets when only the anti-MHC sites are occupied. This is in conflict with the basic findings of MHC-restriction. This problem, which has also been alluded to by Blanden and Ashman (44) and by Mitchison (45), applies not only to Parham's model but to dual recognition models in general. 


\section{The Neoparatope Problem}

Before attempting to provide a solution to the dual recognition problem, it is worthwhile to consider another difficulty that is presented by the approach of Parham (43). In his argument he chooses an antibody that will not bind unless it is bivalent. There, of course, exists within the Bcell repertoire a population of antibodies that have sufficient affinity to bind $\mathrm{MHC}$ antigens in a monovalent fashion. This point brings up the difficulty of selecting a repertoire of T-cell receptors that have only low affinity for both antigen and self MHC at the monovalent level. Since self MHC is present in the organism, it is possible to imagine that some selection mechanism could delete receptors having a high affinity for self MHC. However, it is difficult to imagine such a mechanism for conventional antigen when such antigen is not usually present in the organism and is, moreover, unpredictable. This problem also arises in the context of the neoparatope model. In particular, it is basic to our model that the putative site for conventional antigen not react with high affinity until this site is altered by simultaneous interaction with MHC. Thus, while neoparatopes may have high affinity for conventional antigen, "old" paratopes must have only low apparent affinity. Moreover, MHC-restriction requires that the universe of neoparatopes not overlap with the universe of "old" paratopes. How can the repertoire be selected to have these properties? This is referred to as the neoparatope problem.

\section{A Possible Solution to the Dual Recognition and Neoparatope Problems}

As a solution to these problems, we propose that there is an internal interaction in the T-cell receptor in which the site for conventional antigen is sequestered as a result of an interaction between this site and the antiMHC site. In short, we propose that one site is specific for the other. We further propose that the anti-A site bears an internal image of the MHC determinant that is recognized by the anti-MHC site. This scheme allows the anti-MHC site to have substantial initial affinity for MHC without leading to adherence to other cells that bear only $\mathrm{MHC}$, since the internal image determinant, which is an integral component of the T-cell receptor, should be an especially effective competitive inhibitor, thereby solving the dual recognition problem. If the antigen-contacting residues associated with the anti-A site are also sequestered as a result of the postulated internal interaction, then a low apparent affinity for conventional antigen alone is expected. Moreover, the set of "old" paratopes, which are essentially nonfunctional, does not overlap with the set of neoparatopes. Thus, the neoparatope problem would also be solved. The postulated internal interaction appears compatible with the allosteric mechanism initially 
proposed. The internal bond between the two sites would be continually forming and breaking, allowing occasional bonds to be formed with MHC antigens on other cells. Interaction with conventional antigen, if present, at the anti-A site would interfere with the re-formation of the internal bond and allow the occurrence of the conformational changes that are assumed to be required for the formation of a high-affinity ternary complex.

\section{Evidence for an Internal Inhibitor}

A prediction of the model described above is that an incomplete T-cell receptor lacking the anti-A site, which bears the internal image of $\mathrm{MHC}$, should show binding to self MHC on other cells. Data that are consistent with this possibility have been obtained by Thomas and Hoffman (46). These investigators found that antigen-specific $T$ cells educated in vitro would not adhere to target cells in the absence of specific antigen, as expected. However. if the T cells were lightly trypsinized, adherence to target cells in the absence of antigen was observed. Moreover, the adherence phenomenon showed preference for self MHC. It seems possible that the trypsinization selectively removed the MHC internal image, leaving an incomplete T-cell receptor with an exposed anti-MHC site. With the powerful methods developed recently, it should be possible to carry out a rigorous exploration of these findings.

\section{Evidence for Internal Images of MHC Determinants on T-Cell Receptors}

Sim and co-workers, on the basis of a different theoretical argument, have been led to expect the existence of MHC internal images on T-cell receptors (47). They have provided evidence that monoclonal anti-Class II MHC antibodies as well as cytotoxic T cells specific for Class Il MHC can react with Class Il-restricted $\mathrm{T}$ cells that appear to bear no conventional Class II MHC antigens (47).

It should be emphasized that the concept of a neoparatope implies the existence of neoidiotopes and neointernal images. Therefore, if conformational changes occur upon the formation of a ternary complex, the ability to detect various idiotopes and internal images on T-cell receptors may be dependent on the occupation, or nonoccupation, of the sites for MHC and conventional antigen. This may complicate the detection of internal images on T-cell receptors.

The interpretation of MHC-restriction offered here depends on the existence of internal images of MHC determinants, which may be elements of an idiotypic network involving T-T interactions, as Müllbacher (48) and Sim and co-workers (47) have suggested. The receptor itself can be re- 
garded as a composite structure which contains both Id (anti-MHC) and anti-ld (MHC internal image). It may seem unusual to have these structures in the same receptor (or receptor complex), since they are traditionally regarded as separate molecules in the B-cell compartment. However, the more recent epibody concept is quite reminiscent of what is proposed here for the T-cell receptor. Indeed, the T-cell receptor we envision can be regarded as an "introverted" epibody.

\section{Implications of Antigen Processing for Network Interactions}

According to a currently emerging paradigm (49), antigen recognition by Class II-restricted $T$ cells is dependent upon the processing of conventional antigen by "antigen-presenting" cells. Antigen is internalized by the antigen-presenting cell and subjected to limited proteolytic degradation. Proteolytic cleavage leads to an unfolding of the resulting peptides and the possible exposure of sites preferentially recognized by $\mathrm{T}$ cells $(50)$. Presenting cells pulsed with antigen for a few hours and thoroughly washed continue to present antigen for several days (49). This has recently led to the hypothesis that processed antigen is modified in some way that leads to a firm anchoring in the cell membrane (49). This anchoring is thought to be independent of any interaction between processed antigen and MHC, which is much too weak to account for the stable association with the cell membrane (5I).

Studies by Kakiuchi et al. (52), Rock et al. (53), and Lanzavecchia $(54,55)$ indicate that in the case of B cells, antigen presentation is greatly enhanced when the presenting $B$ cell possesses a membrane antibody that is specific for the antigen. Using a B-cell line transformed by Epstein-Barr virus, Lanzavecchia has offered evidence that tetanus toxoid at a concentration of $10^{-13} \mathrm{M}$ could be presented to cloned $\mathrm{T}$ cells when the B-cell antigen receptor possessed a binding constant of $10^{8}$. B-cell lines not specific for tetanus toxoid could also present this antigen, but required antigen concentrations four orders of magnitude greater. The ability to present antigen that is present in native form at such low concentration would seem to depend on an antigen-specific "pumping" mechanism for internalization as well as firm anchoring of processed antigen in the membrane (56).

This model of antigen presentation represents an evolution of the previous antigen-bridging model of T-B cooperation which envisioned the recognition of native antigen by the $\mathrm{T}$-helper cell (57). As will become evident, this new model of antigen presentation has far-reaching implications for network theories of immune regulation.

Chesnut and Grey demonstrated that B cells can present rabbit antimouse Ig antibodies to $\mathrm{T}$ cells specific for the processed $\mathrm{Fc}$ regions of 
these rabbit antibodies (58). If $\mathrm{B}$ cells can present the $\mathrm{Fc}$ region, it is reasonable to expect that they can also present the v-regions. If the $\mathrm{v}$ regions of the exogenous antibody can be presented, it is likewise reasonable to expect that the endogenously synthesized $v$-regions can be presented, since both sets of $\mathrm{v}$-regions will be internalized as an immune complex.

\section{Mechanistic Basis of the Principle of Symmetrical Stimulation}

The principle of symmetrical stimulation was previously stated as a formal principle. A mechanistic rationalization is required. Consider the immune response to a thymus-dependent protein antigen. The bulk of available data suggests that the antigen is first taken up by an antigen-presenting cell which has no intrinsic antigen specificity, such as a macrophage (59). The efficiency of uptake may be greatly enhanced by the presence of natural antibody (60). After processing, fragments of the antigen are presented on the cell membrane in association with Class II MHC. This leads to activation of T-helper cells. Antigen is also presented by antigen-specific $B$ cells (58). However, the low precursor frequency of antigen-specific B cells suggests that they do not play a role in the initial activation of $T$ cells. But once $T$ cells are activated, they can deliver signals to B cells presenting the appropriate antigen, leading to clonal expansion and highrate antibody secretion. The principle of symmetrical stimulation states that when Id B cells are stimulated, auto-anti-Id B cells are also stimulated. Experimental evidence for auto-anti-Ids is sparse, but most studies have depended on serological methods (61). Very recent studies using monoclonal antibody technology suggest that auto-anti-Ids are readily detectable (62-66). What is the pathway of stimulation? One possibility is that the internalization of antigen by the Id B cells also leads to the presentation of Id v-regions. This could, in turn, lead to the activation of T-helper cells specific for processed Id. These T-helper cells would also encounter processed Id on anti-Id B cells that have interacted with secreted Id. This interaction should stimulate the anti-Id $B$ cells and lead to the secretion of auto-anti-Ids. In addition, interaction of soluble Id with an anti-Id B cell should induce the presentation of the endogenous anti-Id, leading to activation of a population of $\mathrm{T}$ cells specific for processed anti-Id. This pathway of stimulation could, in principle, continue indefinitely. However, the establishment may play a role in truncating idiotypic cascades. The important point here is that when soluble Id interacts with an anti-Id B cell, or when soluble anti-Id interacts with an Id B cell, a T cell specific for either processed Id or anti-Id will activate both specificities of B cells. Thus, there is a symmetry in the stimulation of Id-anti-Id pairs of B-cell clones by $\mathrm{v}$-region specific T-helper cells, as Leserman has also appre- 
ciated (67). Moreover, this stimulation is antibody dependent. This mechanism is offered as the basis of the principle of symmetrical stimulation.

There is another aspect of the principle of symmetrical stimulation which can be rationalized in the context of the new model of antigen presentation. An essential aspect of this principle is that stimulation of $B$ cells by soluble Id or anti-Id can occur at concentrations below the levels at which antibody-mediated cytotoxicity occurs. As noted above, antigen presentation can occur when the antigen concentration is very low. For example, a Bcell receptor having a binding constant of $10^{8}$ could facilitate antigen internalization when the external concentration was $10^{-13} \mathrm{M}$ (55). Presumably, if the binding constant were $10^{10}$, presentation could occur at $10^{-15}$ $M$. Given the bivalency of IgG and the higher valency of secreted IgM, and the high concentration of mobile bivalent membrane receptors on $\mathrm{B}$ cells, it is likely that Id-anti-Id reactions at B-cell surfaces are characterized by high avidity constants. It should be noted that $10^{-15} \mathrm{M} \mathrm{IgG}$ is equivalent to $1.6 \times 10^{-13} \mathrm{~g} \mathrm{ml}^{-1}$. These considerations are offered in support of our initial assumption that low concentrations of Id and anti-Id will lead to stimulation by T-helper cells without generation of significant antibodymediated cytotoxic effects. At much higher concentrations, we expect the occurrence of cytotoxic effects mediated by Fc-receptor-bearing cells (8) and possibly by Class I- or Class II-restricted [(68) and references therein] $T$ cells activated to mediate cytolytic effector functions. The differential effects for high and low concentrations of Id or anti-Id that we have postulated are in line with experimental observations showing that low doses of passively transferred anti-ld tend to activate whereas high doses tend to suppress Id expression, following challenge by antigen (69).

Since the mechanism of symmetrical stimulation is antibody dependent, it is necessary to consider the possible breaking of symmetry by S-antigens in cases where Id or anti-Id has an anti-S specificity. It will be recalled that the breaking of symmetry by S-antigens is a crucial factor in the antagonism of Id-anti-Id pairs. The fact that antigen presentation can take place at extremely low concentrations suggests that $\mathrm{S}$-antigens may not break symmetry, since it is unlikely that adsorption would be efficient enough to reduce levels of Id or anti-Id below the threshold for presentation. Moreover, the threshold for presentation is likely to be several orders of magnitude below the threshold for cytotoxicity effects. Within this window, antigen presentation may not be a sensitive function of concentration, making partial adsorption of Id or anti-Id by S-antigens unimportant. In addition, Id-anti-ld, or S-antigen-Id, immune complexes may be taken up by macrophages and presented. The ability of these complexes to lead to presentation is in contrast with their inability to mediate cytotoxic effects relevant to Id-anti-Id conflicts. Hence, the effects of S-antigen adsorption on stimulation are basically different from the effects on antagonism.

Studies by Kohler and co-workers with the phosphorylcholine (PC) 
system have produced findings that are consistent with processing and presentation of v-regions. It was found that immunization with PC-hemocyanin induces Id-specific T-helper cells that can be detected by their ability to help TNP-specific B cells when challenged with TNP-T15, where $\mathrm{T} 15$ is a $\mathrm{PC}$-specific myeloma protein that bears the idiotype that dominates the response to $\mathrm{PC}$ in $\mathrm{BALB} / \mathrm{c}$ mice (70). This finding is consistent with our prediction that interaction of antigen with specific $B$ cells leads to the presentation of $\mathrm{B}$-cell receptor $\mathrm{v}$-regions and the induction of $\mathrm{v}$-regionspecific T-helper cells.

The population of $\mathrm{T}$-helper cells recognizing the $\mathrm{T} 15$ myeloma protein also recognizes the M167 myeloma protein as highly cross-reactive. M167 is also specific for PC (71). Inhibition experiments suggested that the recognized determinants are heavy-chain associated and are preserved on isolated chains (72). This cross-reactivity in T-cell recognition is in contrast to what is seen with anti-idiotypic antibodies, which generally do not detect cross-reactions with T15 and M167 (71). This suggests that at the level of native immunogenic idiotopes, these myeloma proteins are not similar. However, the cross-reactivity seen by $\mathrm{T}$ cells is consistent with the possibility that these cells recognize processed heavy-chain peptides. The heavy chains from these proteins are identical in the first hypervariable region and differ by only 14 of the first 125 amino acids (21). Depending on cleavage points, peptides of identical sequence could be obtained from both heavy chains, and complete identity may not be needed for crossreactivity.

The results of Jorgensen and Hannestad (73) are also consistent with $v$-region processing. For example, these investigators demonstrated that reduced and fully alkylated $\mathrm{v}$-domains primed $\mathrm{T}$-helper cells as efficiently as native domains when the response to the native domain was monitored.

Rubinstein et al. have observed that injection of $10 \mu \mathrm{g}$ of A48Id into $\mathrm{BALB} / \mathrm{c}$ neonates leads to activation and dominance of the A48Id component of the response to bacterial levan $(74,75)$. In unmanipulated mice this Id is normally absent. Presentation of A48Id v-regions may be involved in the activation process. Indeed, it was found that A48Id dominance was associated with the activation of T-helper cells specific for A48Id $(74,75)$.

\section{Residue Epitopes, Residue Idiotopes, and Residue Internal Images}

The possibility that immunoglobulin v-regions may be processed and presented suggests the existence of an additional dimension of network regulation. Let us consider classical idiotopes on native immunoglobulin v- 
regions. Using the language of Kunkel (10), these antigenic determinants are "individual antigenic specificities" that are associated with unique aspects of the v-region sequences. This same concept can be enlarged to include unique epitopes, i.e., idiotopes, that will likely be present on the processed v-region peptides. Since these peptides represent the residue that remains after partial proteolytic degradation, we refer to these idiotopes as "residue idiotopes." It must, of course, be kept in mind that they are recognized in association with MHC antigens. In this context, an interesting possibility presents itself. Since the set of $v$-region peptides that can be presented by $B$ cells is a diverse set, it is possible that peptides in this set will be cross-reactive with peptides associated with processed conventional antigens. Thus, residue epitopes on processed conventional antigens may cross-react with residue idiotopes. In particular, if a polyclonal population of $\mathrm{T}$ cells specific for a residue epitope also react with a residue idiotope, then the residue idiotope can be regarded as a residue internal image.

The possible existence of these residue elements suggests the existence of a new category of network interactions. It is now necessary to consider that some antibodies may bear both native and residue internal images. Consider a hapten-carrier conjugate, such as adenosine-bovine albumin. There may exist antibodies that in their native state bear internal images of adenosine (62) and in their processed state bear residue internal images of "carrier determinants," i.e., residue images of albumin residue epitopes.

These two-level internal images may be of considerable importance for the development of idiotype vaccines, as they would facilitate the induction of both T-cell and B-cell immunity to a viral protein. Noseworthy et al. may have isolated a monoclonal antibody of this type (76). Their antibody appears to bear an internal image of the reovirus determinant which interacts with the virus receptor on infectable cells (76). Interestingly, immunization with this antibody not only induces antibodies that recognize native virus, but also induces delayed-type hypersensitivity to the virus (77). The latter reactivity is generally attributed to Class II-restricted T cells that recognize processed antigen (78).

Two-level internal images may arise when there is sequence homology between the viral protein and one of the v-regions of the internal image antibody. In such a case, the homologous sequence might lead to native sequence determinants that are cross-reactive. The homologous sequence might also lead to residue determinants that are cross-reactive. It should be emphasized that the shape of the residue determinant might be quite different from the native determinant involving the same amino acids. It is interesting to note that the internal image antibody isolated by Bruck et al. (79) has a v-region stretch of amino acids that is homologous to a stretch of the viral sequence. Although two-level internal images may arise as a result of sequence homology, such homology may not always be 
required, given the mimicry of nonproteinaceous substances such as haptens (5) and carbohydrates (80).

The existence of two-level internal images would be of considerable theoretical importance, since it has heretofore not been obvious how the idiotypic network can store the information that a particular hapten is attached to a carrier molecule (7). Such information may also be stored using two-level epibodies. For example, an antibody specific for a native determinant on a protein could also bear a residue idiotope or residue internal image that cross-reacts with a residue epitope of the protein. Residue epibodies might play a role in the generation of auto-anti-Ids, since a carrier-specific T-helper cell specific for the residue epitope could stimulate an anti-Id $\mathrm{B}$ cell presenting the v-region of the residue epibody.

Residue epibodies may be relevant to the interesting data of Kim et al. (81), which reveal that some cloned T-helper lines specific for ovalbumin facilitate a phosphorylcholine response in which the T15 idiotype dominates and others do not. The T15-inducing clone, which is specific for an ovalbumin residue epitope, could also be specific for a T15 residue idiotope. In this case, the T-helper cell would see a greater density of processed antigen on B cells expressing the T15 Id, which may lead to a quantitatively greater response. This interpretation should be readily tested using synthetic peptides based on the T15 sequence and the existing Tcell clones.

A consideration of network interactions involving residue elements immediately leads to the following question: Is the set of residue idiotopes complete? Another question also arises: Is the set of T-cell receptors that recognize residue elements complete? While these questions cannot, of course, be answered on the basis of available data, some considerations can be made.

The concept of completeness is intimately related to the concept of specificity. On the basis of considerable data, it is clear that a single antibody molecule can exhibit multispecificity in antigen binding (82). This means that antibodies do not have absolute powers of discrimination. Clearly, the greater the degree of multispecificity, the greater the likelihood of interactions within the B-cell repertoire.

These considerations can be transferred to the residue compartment. The first point to be noted is that the number of native antibody v-regions is likely to be greater than the number of v-region peptides that can be presented as processed fragments. On the other hand, there is evidence that $T$ cells exhibit a higher degree of multispecificity than B cells [(24) and references therein]. This could compensate for the smaller universe of processed v-region peptides and allow for a functional network involving residue elements. However, it should be noted that the findings of Jorgensen and Hannestad suggest that Ir gene defects may place some restrictions on completeness (73). 


\section{IgD May Play a Special Role in the Presentation of Endogenous v-Regions}

An especially puzzling feature of B cells is the existence of two isotypes of membrane receptors. In addition to monomeric IgM, there is also an $\operatorname{IgD}$ receptor (9). The function of the $\operatorname{IgD}$ isotype, which occurs almost entirely as a membrane receptor (83), has continued to resist clarification. A unique feature of $\operatorname{IgD}$ is the proteolytic lability of its hinge and Fab regions (84). Also intriguing is the fact that immature $B$ cells have only IgM (9). From the perspective generated by this discussion, an interesting possibility for IgD function presents itself. An essential step in antigen presentation is the proteolytic cleavage of the antigen into fragments. The greater lability of $\mathrm{IgD}$ hinge and Fab regions suggests that they may be processed more efficiently, leading to a greater concentration of processed fragments on the membrane and a greater potential for the stimulation of residue-idiotope-specific T-helper cells. That this would occur on mature $\mathrm{B}$ cells and not immature cells is interesting. Conceivably, immature B cells expressing inappropriate $v$-regions might first be subjected to negative selection before the expression of IgD occurs.

In this discussion, antigen presentation has been viewed as a process in which molecules in the extracellular fluid or on the cell membrane are internalized. Very recent evidence suggests that internally synthesized proteins can also be presented $(68,85)$. This possibility makes it important to emphasize that an assumption of the model presented here is that $\mathrm{v}$ regions of $\mathrm{B}$ cells unstimulated by antigen or anti-Id are not presented. It is assumed that antigen processing takes place in specialized compartments and that access to these compartments is a controlled process, as some experimental evidence is already beginning to indicate $(86,87)$.

The model of v-region processing offered here differs from prior suggestions in several important features. A recent model, proposed by Leserman (67), appears heavily influenced by the determinant selection hypothesis (88). This hypothesis assumes that processed antigen adheres to the membrane of an antigen-presenting cell because of its affinity for Class II MHC. Leserman notes the difficulty of understanding how the myriad of conventional antigens can all have affinity for the several MHC molecules present in a single individual. He then assumes that presentation of conventional antigen is an incidental, occasional feature and that a uniform and centrally important consequence of the interaction of antigen with $\mathrm{B}$-cell receptors is to induce the presentation of the B-cell receptors. $\mathrm{He}$ envisions that presentation involves antigen-induced endocytosis and limited proteolysis to expose an invariant hydrophobic site, which may be located in $\mathrm{CH}$ l, and which has affinity for Class II MHC molecules. Return of the processed Ig to the cell surface leads to Ig-MHC complex formation and subsequent $\mathrm{T}$-cell recognition. The important point here is that the invariant site must remain connected to the hypervariable region 
where idiotypic determinants are located. This means that proteolysis must be relatively limited.

The model offered in this discussion assumes that membrane anchorage of processed Ig is independent of any affinity for MHC, although weak affinity may exist (51) and may play a role in the formation of the ternary complex with the T-cell receptor. The mechanism for membrane anchorage is assumed to be similar for both conventional antigen and Ig. Hence, presentation of conventional antigen and of $\mathrm{Ig}$ are of equal likelihood and importance. Moreover, the anchoring mechanism, which may involve the formation of covalent bonds, imposes no currently apparent restriction on the degree of proteolysis. On the basis of experiments with synthetic peptides (89), processed peptides may be as short as about 15 amino acids.

McMamara et al. (72), on the basis of their data and those of Jorgensen and Hannestad (73), have suggested that T cells "see" individual Ig chains and that antigen-presenting cells may separate Ig molecules into free polypeptides. This suggestion also envisions very limited modifications.

If we are correct in suggesting that processing for $v$-regions is similar to that for conventional antigens, then an experimental exploration of $\mathrm{v}$ region processing is immediately accessible, given the availability of known $v$-region sequences for some antigen systems, the availability of T-cell clones and hybridomas, and the ease with which short peptides can be synthesized.

\section{A Possible Mechanism for Network Control of B-Cell and T-Cell Rearrangements}

Complete immunoglobulin and T-cell receptor genes are assembled by rearrangement mechanisms in which germline gene segments are selected and joined to form complete genes. To assemble, for example, an immunoglobulin heavy-chain variable region, recombination between three different germline elements $(\mathrm{V}[\mathrm{H}], \mathrm{D}, \mathrm{J})$ must occur. In the mouse, the $\mathrm{v}[\mathrm{H}]$ segment must be selected from a set that contains 200 to 1,000 elements. The D and $J$ sets contain 12 and 4 elements, respectively (9). As yet, a detailed understanding of the forces that regulate these recombination events has not been achieved. However, Yancopoulos and Alt have proposed a model which suggests that a single recombinase mediates the assembly of all Ig and T-cell receptor variable gene segments (90). An essential aspect of this model is that for the recombinase to be active on a particular gene segment, the gene segment must be exposed. They have observed that germline $\mathrm{V}[\mathrm{H}]$ segments are transcriptionally active before rearrangement. They assume that this transcriptional activity ensures the exposure necessary for recombinase activity.

It is proposed here to extend the accessibility model of Yancopoulos and Alt to include a mechanism by which $\mathrm{T}$ cells can influence the selection 
of $\mathrm{V}[\mathrm{H}]$ segments by the recombinase. Essentially three additional assumptions are needed for this extension.

Antigen presentation is usually visualized as a process in which proteins in the external fluid are internalized and then processed. Previously in this discussion, it was also assumed that endogenously synthesized membrane immunoglobulins can be internalized and processed when they interact with antigens or antibodies. In the context of the Jancopoulos-Alt model, we further assume that $\mathrm{V}[\mathrm{H}]$ transcripts produce peptides which find their way into the compartments where the processing required for antigen presentation takes place. Although this could occur by an exogenous pathway in which peptides having leader sequences are brought to the cell surface and then reinternalized, it is of special interest to consider the possible existence of an endogenous pathway for antigen presentation. Evidence for this kind of pathway has recently been obtained with internal viral proteins that have no leader sequences. Recent evidence suggests that cytolytic $T$ cells can kill infected cells through recognition of processed fragments of internal viral proteins $(68,85)$.

The second assumption is that the transcription of unrearranged $\mathrm{V}[\mathrm{H}]$ segments is a stochastic process that results in different $\mathrm{V}[\mathrm{H}]$ segments being transcribed in different unrearranged cells at a particular time. Thus, while population studies may suggest transcription of large fraction of $\mathrm{V}[\mathrm{H}]$ segments, we assume that in individual cells, transcription is restricted to a stochastically selected and possibly time-variable subset of $\mathrm{V}[\mathrm{H}]$ segments. This means that different cells will, at a particular point in time, present the processed peptides of different sets of $\mathrm{V}[\mathrm{H}]$ segments. As a result, it becomes possible for Class II-restricted T cells to "know" which $\mathrm{V}[\mathrm{H}]$ segments are transcriptionally active in a particular cell and, therefore, which $\mathrm{V}[\mathrm{H}]$ segments are likely targets of recombinase selection.

If it is further assumed that $\mathrm{T}$ cells specific for residue epitopes of $\mathrm{V}[\mathrm{H}]$ translation products can deliver signals that can activate the recombinase, then a mechanism is established by which $\mathrm{T}$ cells can control or select rearrangements. The specificity of the control will clearly depend on the fraction of $\mathrm{V}[\mathrm{H}]$ segments that are transcribed within the time interval required for $\mathrm{T}$-cell recognition and activation of the recombinase. Conceivably, the effect of this control may be to focus the recombinase on a particular $\mathrm{V}[\mathrm{H}]$ subfamily, rather than to select an individual $\mathrm{V}[\mathrm{H}]$ segment. The sequence homology that would exist within a $\mathrm{V}[\mathrm{H}]$ family or subfamily (9l) should yield frequent cross-reactions among processed peptides from $\mathrm{V}[\mathrm{H}]$ segments belonging to that subfamily.

This model can be extended to include $\mathrm{DJ}[\mathrm{H}]$ segments which are transcriptionally active and which lead to $D[\mu]$ chains that contain 20 to 40 amino acids and also appear to contain a hydrophobic leaderlike sequence (9). Control of class switching by $T$ cells specific for residue epitopes on processed translation products of unrearranged constant-region gene seg- 
ments might also be possible, since these gene segments remain transcriptionally active after the formation of a $\mu$-chain gene $(9,92)$.

In this model, it has been assumed that the $\mathrm{T}$ cell activates the recombinase. The $\mathrm{T}$ cell in question might be a Class II-restricted T-helper that secretes the lymphokines appropriate for recombinase activation. It should be noted that there is already in vitro evidence that $\mathrm{T}$ cells together with dendritic cells can drive the maturation of B-lineage cells (92). However, it seems prudent to be open to the possibility of negative selection by Class I- or Class II-restricted CTL. Pre-B cells are reported to express Class II MHC antigens (93) and presumably express Class I MHC antigens.

As yet there is little evidence that transcripts of unrearranged $\mathrm{V}[\mathrm{H}]$ segments are translated. However, Ucker and co-workers have recently discovered a cytotoxic T-cell line in which an unrearranged $\mathrm{V}[\mathrm{H}]$ gene is transcribed and translated (94). Interestingly, the product appears to be on the cell membrane. From a conventional point of view, this is puzzling, since the peptide possesses no membrane-anchoring sequence. The authors suggest a noncovalent association with the heterodimeric $\mathrm{T}$-cell receptor. Alt and co-workers have offered the similar suggestion that the sequence homology between $\mathrm{V}[\mathrm{H}]$ segments and $\beta 2$-microglobulin may allow binding to Class I MHC chains (9). Our suggestion that translation products of $\mathrm{V}[\mathrm{H}]$ segments can be processed represents an alternative basis for the membrane association of the $\mathrm{V}[\mathrm{H}]$ peptide described by Ucker and coworkers. However, it should be noted that processing of $\mathrm{V}[\mathrm{H}]$ peptides is assumed to be controlled so that it normally occurs before rearrangement and not after. The $\mathrm{V}[\mathrm{H}]$ expression observed by Ucker et al. may therefore be aberrant.

If it is granted that the T-cell repertoire is shaped by network interactions, then this model provides for network control of immunoglobulin and possibly $\mathrm{T}$-cell receptor rearrangements. It is relevant to consider if this model makes sense in the context of network regulation. One consequence of T-cell selection of gene segments would be to ensure that the $v$-region peptides generated after antigen recognition can be recognized by the available T-cell repertoire. Except for differences due to junctional diversity and somatic mutation (9), peptides from complete v-regions should be similar to peptides from unrearranged segments. The findings of Jorgensen and Hannestad (73) demonstrate Ir gene defects in the recognition of syngeneic v-regions. Such defects may be minimized by the rearrangement control mechanism offered here.

\section{Conclusion}

Although incomplete in many respects, the model offered in this discussion is, nonetheless, sufficiently well elaborated to allow many testable predictions, only a few of which have been described because of space lim- 
itations. These predictions are of importance not only to basic questions of immune regulation but also to the practical use of monoclonal antibodies in the treatment of cancer and autoimmune diseases and as idiotype vaccines. It is hoped that the perspective generated by this model will inspire the design of experiments that will lead to the direct evidence that is needed to prove or disprove the existence of an idiotypic network.

Acknowledgments. Bernard F. Erlanger is thanked for his support and encouragement and for many stimulating discussions concerning subjects discussed in this paper. R. G. Miller, M. J. Bevan, and T. J. Braciale are also thanked for helpful discussions. This work was supported by the Evans Foundation.

\section{References}

1. Burnet, F.M. 1957. A modification of Jerne's theory of antibody using the concept of clonal selection. Aust. Sci. 20:67.

2. Talmage, D.W. 1957. Allergy and immunology. Annu. Rev. Med. 8:239.

3. Jerne, N.K. 1974. Towards a network theory of the immune response. Ann. Immunol. (Inst. Pasteur) 125C:373.

4. Paul, W.E.. and C. Bona. 1982. Regulatory idiotopes and immune networks: a hypothesis. Immunol. Today 3:230.

5. Farid, N.R., and T. C.Y. Low. 1985. Anti-idiotypic antibodies as probes for receptor structure and function. Endocr. Rev. 6:1.

6. Ehrlich, P. 1900. On immunity with special reference to cell life. Proc. Roy. Soc., Ser. B. 66:424.

7. Cohn, M. 1981. Conversations with Niels Kaj Jerne on immune regulation: associative versus network recognition. Cell. Immunol. 61:425.

8. Lanier, L.L., and J.H. Phillips. 1986. Evidence for three types of human cytotoxic lymphocyte. Immunol. Today 7:132.

9. Alt, F.W., T.K. Blackwell, R.A. DePinho, M.G. Reth, and G.D. Yancopoulos. 1986. Regulation of genome rearrangement events during lymphocyte differentiation. Immunol. Rev. 89:5.

10. Kunkel, H.G. 1970. Experimental approaches to homogeneous antibody populations. Individual antigenic specificity, cross-specificity and diversity of human antibodies. Fed. Proc. 29:55.

11. Nisonoff, A., S-T. Ju, and F. Owen. 1977. Studies of structure and immunosuppression of a cross-reactive idiotype in strain A mice. Immunol. Rev. 34:89.

12. Brient, B.W., and A. Nisonoff. 1970. Quantitative investigations of idiotypic antibodies. IV. Inhibition by specific haptens of the reaction of anti-hapten antibody with its anti-idiotypic antibody. J. Exp. Med. 132:951.

13. Jerne, N.K., J. Roland, and P.-A. Casenave. 1982. Recurrent idiotypes and internal images. EMBO J, 1:243.

14. Bona, C.. S. Finley, S. Waters, and H.G. Kunkel. Anti-immunoglobulin antibodies. III. Properties of sequential anti-idiotypic antibodies to heterologous anti-gamma globulins. Detection of reactivity of anti-idiotype antibodies with 
epitopes of Fc fragments (homobodies) and with epitopes and idiotypes (epibodies). J. Exp. Med. 156:986.

15. Holmberg, D., S. Forsgren, L. Forni, F. Ivars, and A. Coutinho. 1984. Idiotypic determinants of natural IgM antibodies that resemble self Ia antigens. Proc. Natl. Acad. Sci. USA. 81:3175.

16. Jerne, N.K. 1984. Idiotypic networks and other preconceived ideas. Immunol. Rev. 79:5.

17. Erlanger, B.F. 1985. Anti-idiotypic antibodies: what do they recognize? Immunol. Today 6:10.

18. Wassermann, N.H., A.S. Penn, P.I. Freimuth, N. Treptow, S. Wentzel, W.L. Cleveland, and B.F. Erlanger. 1982. Anti-idiotypic route to anti-acetylcholine receptor antibodies and experimental myasthenia gravis. Proc. Natl. Acad. Sci. USA 79:4810.

19. Tzartos, S.J., M.E. Seybold, and J.M. Lindstrom. 1982. Specificities of antibodies to acetycholine receptors in sera from myasthenia gravis patients measured by monoclonal antibodies. Proc. Natl. Acad. Sci. USA 79:188.

20. Cunningham, A.J. 1974. The generation of antibody diversity: its dependence on antigenic stimulation. Contemp. Top. Mol. Immunol. 3:1.

21. Gearhart, P.J., N.D. Johnson, R. Douglits, and L. Hood. 1981. IgG antibodies to phosphorylcholine exhibit more diversity than their IgM counterparts. Nalure (London) 291:29.

22. Manser, T., L.J. Wysocki, T. Gridley, R.I. Near, and M.L. Gefter. 1985. The molecular evolution of the immune response. Immunol. Today 6:94.

23. Jerne, N.K. 1971. The somatic generation of immune recognition. Eur. J. Immunol. 1:1.

24. Cleveland, W.L., and B.F. Erlanger. 1984. Hypothesis: the MHC-restricted T-cell receptor as a structure with two multistate allosteric combining sites. Mol. Immunol. 21:1037.

25. Martinez-A, C., R.R. Bernabé, A. de la Hera, P. Pereira, P-A. Casenave, and A. Coutinho. 1985. Establishment of idiotypic helper T-cell repertoires early in life. Nature (London) 317:721.

26. Staerz, U.D., and M. Bevan. 1986. Use of anti-receptor antibodies to focus T-cell activity. Immunol. Today 7:241.

27. Tite, J.P., J. Kaye, K.M. Saizawa, J. Ming, M.E. Katz, L.A. Smith, and C.A. Janeway. 1986. Direct interactions between B and T lymphocytes bearing complementary receptors. J. Exp. Med. 163:189.

28. Walden, P., Z.A. Nagy, and J. Klein. 1985. Induction of regulatory T-lymphocyte responses by liposomes carrying major histocompatibility complex molecules and foreign antigen. Nature (London) 315:327.

29. Miller, R.G., and H. Derry. 1979. A population in nu/nu spleen can prevent generation of cytotoxic lymphocytes by normal spleen cells against self antigens of the nu/nu spleen. J. Immunol. 122: 1502.

30. Miller, R.G. 1986. The veto phenomenon and T-cell regulation. Immunol. Today 7:112.

31. Claesson, M.H., and R.G. Miller. 1984. Functional heterogeneity in allospecific cytotoxic T lymphocyte clones. I. CTL clones express strong antiself suppressive activity. J. Exp. Med. 160:1702.

32. Fink. P.J., H.-G. Rammensee, J. D. Benedetto. U.D. Staerz, L. Lefrancois, and M.J. Bevan. 1984. Studies on the mechanism of suppression of primary cytotoxic responses by cloned cytotoxic T-lymphocytes. J. Immunol. 133:1769. 
33. Rammensee, H-G., M.J. Bevan, and P. Fink. 1985. Antigen specific suppression of T-cell responses-the veto concept. Immunol. Today. 6:41.

34. Fink, P.J., H-G., Rammensee, J.D. Benedetto, U.D. Staerz, L. Lefrancois, and M.J. Bevan. 1984. Studies on the mechanism of primary cytotoxic responses by cloned cytotoxic T lymphocytes. J. Exp. Med. 133:1769.

35. Araneo, B.A., and R.L. Yowell. 1985. MHC-linked immune response suppression mediated by $\mathrm{T}$-cells bearing $\mathbf{I}$-A-encoded determinants. J. Immunol. 135:73.

36. Goverman, J., T. Hunkapiller, and L. Hood. 1986. A speculative view of the multicomponent nature of $T$ cell antigen recognition. Cell 45:475.

37. Bushkin, Y., D.N. Posnett, B. Pernis, and C.Y. Wang. 1986. A new HLAlinked $\mathrm{T}$ cell membrane molecule related to the $\mathrm{B}$ chain of the clonotypic receptor is associated with T3. J. Exp. Med. 164:458.

38. Bank, I., R.A. DePinho, M.B. Brenner, J. Cassimeris, F.W. Alt, and L. Chess 1986. A functional T3 molecule associated with a novel heterodimer on the surface of immature human thymocytes. Nature (London) 322: 179.

39. Brenner, M.B., J. McLean, D.P. Dialynas, J.L. Strominger, J.A. Smith, F.L. Owen, J.G. Seidman, S. Ip, F. Rosen, and M.S. Krangel. 1986. Identification of a putative second T-cell receptor. Nature (London) 322:145.

40. Hood, L., M. Kronenberg, and T. Hunkapiller. 1985. T-cell antigen receptors and the immunoglobulin supergene family. Cell 40:225.

41. Watts, T.H., H.E. Graub, and H.M. McConnell. 1986. T-cell-mediated association of peptide antigen and major histocompatibility complex protein detected by energy transfer in an evanescent wave-field. Nature (London) 320: 176 .

42. Ashwell, J.O., and R.H. Schwartz. 1986. T-cell recognition of antigen and the Ia molecule as a ternary complex. Nature (London) 320:176.

43. Parham. P. 1984. A repulsive view of MHC restriction. Immunol. Today. 5:89.

44. Blanden, R.V., and R.B. Ashman. 1985. Speculation: Selection of pre-T cells in the thymus by unique combinations of major and minor histocompatibility antigens. Mol. Immunol. 22:827.

45. Mitchison, N.A. 1986. Antigen binding and T-cells. Nature (London) 320:106.

46. Thomas. D.W., and M.D. Hoffman. 1982. Evidence for covert cellular interaction sites expressed by activated T lymphocytes. J. Immunol. 129: 1416.

47. Sim, G.K., I.A. MacNeil, and A.A. Augustin. 1986. T helper cell receptors: idiotypes and repertoire. Immunol. Rev. 90:49.

48. Müllbacher, A. 1981. Natural tolerance: a model for Ir gene effects in the cytotoxic $\mathrm{T}$ cell response to $\mathrm{H}-\mathrm{Y}$. Transplantation 32:58.

49. Benacerraf, B., L.D. Fallow, Jr., and K.L. Rock. 1986. Processing of native antigen by accessory cells and presentation of membrane bound $\mathrm{MHC}$-associated antigen to specific T cells. In B. Pernis, S.C. Silverstein, and H.J. Vogel (eds.), Processing and presentation of antigens. Academic Press, Orlando. Florida. In press.

50. DeLisi, C., and J.A. Berzofsky. 1985. T-cell antigenic sites tend to be amphipathic structures. Proc. Natl. Acad. Sci. USA 82:7048.

5I. Babbitt, B.P., P.M. Allen, G. Matsueda, E. Haber. and E. R. Unanue. 1985 Binding of immunogenic peptides to la histocompatibility molecules. Nature (London) 317:359. 
52. Kakiuchi, T., R.W. Chesnut, and H. Grey. 1983. B-cells as antigen-presenting cells: the requirement for B-cell activation. J. Immunol. 131:109.

53. Rock, K.I., B. Benacerraf, and A.K. Abbas. 1984. Antigen presentation by hapten-specific B lymphocytes. I. Role of surface immunoglobulin receptors. J. Exp. Med. 160:1102.

54. Lanzavecchia, A. 1985. Antigen-specific interaction between $\mathrm{T}$ and $\mathrm{B}$ cells. Nature (London) 314:537.

55. Lanzavecchia, A., S. Siervo, and D. Scheidegger. 1986. On the role of B-cell surface Ig in antigen presentation to T-cells. In B. Pernis, S.C. Silverstein, and H.J. Vogel, (eds.), Processing and presentation of antigens. Academic Press, Orlando, Florida. In press.

56. Howard, J.C. 1985. Immunological help at last. Nature (London) 314:495.

57. Mitchison, N.A. 1971. The carrier effect in the secondary response to haptenprotein conjugates. II. Cellular cooperation. Eur. J. Immunol. 1:18.

58. Chesnut, R., and H. Grey. 1981. Studies on the capacity of B cells to serve as antigen-presenting cells. J. Immunol. 126:1075.

59. Unanue, E.R. 1984. Antigen-presenting function of the macrophage. Annu. Rev. Immunol. 2:395.

60. Celis, E., and T.W. Chang. 1984. Antibodies to hepatitis B surface antigen potentiate the response of human $\mathrm{T}$ lymphocyte clones to the same antigen. Science 224:297.

61. Rodkey, L.S. 1980. Autoregulation of immune responses via idiotype network interactions. Microbiol. Rev. 44:631.

62. Shechter, Y., D. Elias, R. Bruck, R. Maron, and I. Cohen. Mice immunized to insulin develop anti-idiotypic antibody to the insulin receptor. This volume. Ch. 8.

63. Cleveland, W.L., N.H. Wasserman, R. Sarangarajan, A.S. Penn, and B.F. Erlanger. 1983. Monoclonal antibodies to the acetylcholine receptor (AChR) by a normally functioning auto-anti-idiotypic mechanism. Nature (London) 305:56.

64. Cayanis, E., R. Rajagopalan, W.L. Cleveland, I.S. Edelman, and B.F. Erlanger. 1986. Generation of an auto-anti-idiotypic antibody that binds to glucocorticoid receptor. J. Biol. Chem. 261:5094.

65. Ku, H-h., W.L. Cleveland, and B.F. Erlanger. Adenosine receptors: an autoanti-idiotypic approach. Submitted for publication.

66. Cleveland, W.L., and B.F. Erlanger. 1986. The auto-anti-idiotypic strategy for preparing monoclonal antibodies to receptors. Methods Enzymol. 121: 95.

67. Leserman, L. 1986. The introversion of the immune response: a hypothesis for T-B interaction. Immunol. Today 6:352.

68. Morrison, L.A., A.E. Lukacher, V.L. Braciale, D.P. Fan, and T.J. Braciale. 1986. Differences in antigen presentation to MHC Class I- and Class 11-restricted influenza virus-specific cytolytic clones. J. Exp. Med. 163:903.

69. Reth, M., G. Kelsoe, and K. Rajewsky. 1981. Idiotypic regulation by isologous monoclonal anti-idiotype antibodies. Nature (London) 290:257.

70. Gleason, K., S. Pierce, and H. Kohler. 1981. Generation of idiotype-specific T cell help through network perturbation. J. Exp. Med. 153:924.

71. McNamara, M., and H. Kohler. 1983. Idiotype-recognizing T helper cells that are not idiotype specific. J. Exp. Med. 158:81I. 
72. McNamara. M., K. Gleason, and H. Kohler. 1984. T-cell helper circuits. Immunol. Rev. 79:87.

73. Jorgensen, T., and K. Hannestad. 1982. Helper T' cell recognition of the variable domains of a mouse myeloma protein $(315)$ : effect of the major histocompatibility complex and domain conformation. J. Exp. Med. 155:1587.

74. Rubinstein, L.J., and C. Bona. 1983. Idiotype-anti-idiotype network. III. Genetic control of activation of A48Id silent clones subsequent to manipulation of the immune network. Ann. N.Y. Acad. Sci. 418:97.

75. Bona, C.A., C. Victor-Kobrin, A.J. Manheimer, B. Bellon, and L.J. Rubinstein. 1984. Regulatory arms of the immune network. Immunol. Rev. 79:25.

76. Noseworthy, J.H., B.N. Fields, M.S. Dichter, C. Sobotka. E. Pizer, L.I. Perry. J.T. Nepom, and M.I. Greene. 1983. Cell receptors for the mammalian reovirus. I. Syngeneic monoclonal anti-idiotypic antibody identifies a cell surface receptor for reovirus. J. Immunol. 131:2533.

77. Sharpe, A.H., G.N. Gaulton, K.K. McDade, B.N. Fields, and M.l. Greene. 1984. Syngeneic monoclonal antiidiotype can induce cellular immunity to reovirus. J. Exp. Med. 160: 1195.

78. Miller. J.F.A.P., M.A. Vadas, A. Whitelaw, and J. Gamble. 1975. H-2 gene complex restricts transfer of delayed-type hypersensitivity in mice. Proc. Natl. Acad. Sci. USA 72:5095.

79. Bruck, C., M.S. Co., M. Slaoui, G.N. Gaulton, T. Smith, B.N. Fields, J.I. Mullins, and M.I. Greene. 1986. Nucleic acid sequence of an internal imagebearing monoclonal anti-idiotype and its comparison to the sequence of the external antigen. Proc. Natl. Acad. Sci USA. 83:6578.

80. Rubinstein. L.J.. B. Goldberg, J. Hiernaux. K.E. Stein, and C. Bona. 1983. ldiotype-antiidiotype regulation. $\mathrm{V}$. The requirement for immunization with antigen or monoclonal antiidiotypic antibodies for the activation of $\beta 2 \rightarrow 6$ and $\beta 2 \rightarrow 1$ polyfructosan-reactive clones in $B A L B / c$ mice treated at birth with minute amounts of anti-A48 idiotype antibodies. J. Exp. Med. 158:1129.

8I. Kim, J.. A. Woods, E. Becker-Dunn, and K. Bottomly. 1985. Distinct functional phenotypes of cloned la-restricted helper T cells. J. Exp. Med. 162:188.

82. Ghosh. S., and A.M. Campbell. 1986. Multispecific monoclonal antibodies. Immunol. Today 7:217.

83. Rowe, D.S., K. Hug, L. Forni, and B. Pernis, 1973. Immunoglobulin D as a lymphocyte receptor. J. Exp. Med. 138:965.

84. Spiegelberg, H.L. 1977. The structure and biology of human IgD. Immunol. Rev. 37:3.

85. Townsend, A.R.M., J. Rothbard, F.M. Gotch, G. Bahadur, D. Wraith, and A.J. McMichael. 1986. The epitopes of influenza nucleoprotein recognized by cytotoxic T lymphocytes can be defined with short synthetic peptides. Cell 44:959.

86. Pernis. B. 1985. Internalization of lymphocyte membrane components. Immunol. Today 6:45.

87. Ruud, E., H.K. Blomhoff, S. Funderud, and T. Godal. 1986. Internalization and processing of antibodies to surface antigens on human $\mathrm{B}$ cells. Monoclonal anti-lgM antibodies are processed differently than monoclonal antibodies towards non-lg surface receptors. Eur. J. Immunol. 16:286.

88. Schwartz, R.H., E. Heber-Katz, and D. Hansburg. 1983. The la molecule contributes to the specificity of T cell activation. In J.W. Parker and R.L. 
O'Brien (eds.), Intercellular communication in leucocyte function, pp. 117125. John Wiley \& Sons, Ltd., New York.

89. Hansburg, D. E. Heber-Katz, T. Fairwell, and E. Appella. 1983. Major histocompatibility complex $x$ controlled antigen-presenting cell-expressed specificity of T cell antigen recognition. J. Exp. Med. 158:25.

90. Yancopoulos, G.D., and F.W. Alt. 1985. Developmentally controlled and tissue specific expression of unrearranged $\mathrm{V}[\mathrm{H}]$ gene segments. Cell 40:27I.

91. Brodeur, P. H., and R. Rible1. 1984. The immunoglobulin heavy chain variable region (Igh-V) locus in the mouse. I. One hundred Igh- $V$ genes comprise seven families of homologous genes. Eur. J. Immunol. 14:922.

92. Spalding, D.M., and J.A. Griffin. 1986. Different pathways of differentiation of pre-B cell lines are induced by dendritic cells and T-cells from different lymphoid tissues. Cell 44:508.

93. Hokland, P.. J. Ritz, S.F. Schlossman, and L. Nadler. 1985. Orderly expression of $B$ cell antigens during the in vitro differentiation of nonmalignant human pre-B cells. J. Immunol. 135:1746.

94. Ucker, D.S., Y. Kurasawa, and S. Tonegawa. 1985. An unusual clonotypic determinant on a cytotoxic $\mathrm{T}$ lymphocyte line is encoded by an immunoglobulin heavy chain variable region gene. J. Immunol. 135:4204. 